\title{
Chiral SU(3) quark mean-field model for hadronic systems
}

\author{
P. Wang ${ }^{\mathrm{a}}$, D. B. Leinweber ${ }^{\mathrm{a}}$, A. W. Thomas ${ }^{\mathrm{ab}}$ and A. G. Williams ${ }^{\mathrm{a}}$

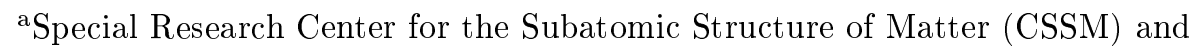 \\ Department of Physics, University of Adelaide 5005, Australia \\ b Jefferson Laboratory, 12000 Jefferson Ave., Newport News, VA 23606 USA
}

We perform a study of infinite hadronic matter and finite nuclei at finite temperature and density with an improved method of calculating the effective baryon mass. A detailed study of the predictions of the model is made in comparison with the available data and the level of agreement is generally very good.

\section{INTRODUCTION}

The complex action of QCD at finite chemical potential makes it difficult to study finitedensity QCD properties directly from first principle lattice calculations. There are many phenomenological models based on hadronic degrees of freedom, such as the Walecka model [1], the Zimanyi-Mozkovski model [2] and the nonlinear $\sigma-\omega$ model [3], as well as models which explicitly include quark degrees of freedom, for example, the quark meson coupling model [4], the cloudy bag model [5], the quark mean-field model [6] and the NJL model [7]. With the development of these phenomenological models, the interactions between hadrons become more and more complex. The symmetries of QCD can be used to determine largely how the hadrons should interact with each other. With this in mind, the chiral $S U(2) \times S U(2)$ effective quark model was proposed. It has been used widely to investigate nuclear matter and finite nuclei at both zero and finite temperature.

Recently, we proposed a chiral $S U(3)$ quark mean-field model based on quark degrees of freedom $[8,9]$. In this model, quarks are confined in the baryons by an effective potential. The quarkmeson interaction and meson self-interaction are based on $S U(3)$ chiral symmetry. Through the mechanism of spontaneous symmetry breaking the resulting constituent quarks and mesons (except for the pseudoscalars) obtain masses. The introduction of an explicit symmetry breaking term in the meson self-interaction generates the masses of the pseudoscalar mesons which satisfy the relevant PCAC relations. The explicit symmetry breaking term in the quark-meson interaction gives reasonable hyperon potentials in hadronic matter. This chiral $S U(3)$ quark meanfield model has been applied to investigate nuclear matter [10], strange hadronic matter [8], finite nuclei, hypernuclei [9], and quark matter [11]. By and large the results are in reasonable agreement with existing experimental data.

A surprising result, which is quite different from most other models is that at some critical density the effective baryon mass drops to zero [12]. There is a first order phase transition around this critical density where the physical quantities change discontinuously. We will see later that this behavior is primarily a consequence of the nonlinear ansatz for the effective baryon mass which is related to the subtraction of the centre of mass (c.m.) motion. In Ref. [13], the authors provided an exact solution of the effect of c.m. motion and found that it was only very weakly dependent on the external field strength for the densities of interest. As a result, the c.m. correction can be included in the zero point energy. In this paper, we will use this alternative definition of the effective baryon mass in medium to reexamine the properties of nuclear systems. A successful model should describe well the properties of nuclear matter, not only at zero temperature but also at finite temperature. We will also apply the chiral $S U(3)$ quark mean-field model to study the 
liquid-gas phase transition and Comloub instability of asymmetric nuclear systems and compare our results with the recent experimental analysis.

The paper is organized as follows. The model is introduced in Sec. II. In Sec. III, we study nuclear systems at finite temperature and density. The numerical results are presented in Sec. IV.

\section{THE MODEL}

Our considerations are based on the chiral $S U(3)$ quark mean-field model (for details see Refs. $[8,9]$ ), which contains quarks and mesons as basic degrees of freedom. In the chiral limit, the quark field $q$ can be split into left and righthanded parts $q_{L}$ and $q_{R}: q=q_{L}+q_{R}$. Under $S U(3)_{L} \times S U(3)_{R}$ they transform as

$q_{L} \rightarrow q_{L}^{\prime}=L q_{L}, \quad q_{R} \rightarrow q_{R}^{\prime}=R q_{R}$.

The spin- 0 mesons are written in the compact form

$M\left(M^{+}\right)=\Sigma \pm i \Pi=\frac{1}{\sqrt{2}} \sum_{a=0}^{8}\left(s^{a} \pm i p^{a}\right) \lambda^{a}$,

where $s^{a}$ and $p^{a}$ are the nonets of scalar and pseudoscalar mesons, respectively, $\lambda^{a}(a=1, \ldots, 8)$ are the Gell-Mann matrices, and $\lambda^{0}=\sqrt{\frac{2}{3}} I$. The alternatives, plus and minus signs correspond to $M$ and $M^{+}$. Under chiral $S U(3)$ transformations, $M$ and $M^{+}$transform as $M \rightarrow M^{\prime}=L M R^{+}$and $M^{+} \rightarrow M^{+^{\prime}}=R M^{+} L^{+}$. The spin-1 mesons are arranged in a similar way as

$l_{\mu}\left(r_{\mu}\right)=\frac{1}{2}\left(V_{\mu} \pm A_{\mu}\right)=\frac{1}{2 \sqrt{2}} \sum_{a=0}^{8}\left(v_{\mu}^{a} \pm a_{\mu}^{a}\right) \lambda^{a}$

with the transformation properties: $l_{\mu} \rightarrow l_{\mu}^{\prime}=$ $L l_{\mu} L^{+}, r_{\mu} \rightarrow r_{\mu}^{\prime}=R r_{\mu} R^{+}$. The total effective Lagrangian is written:

$$
\begin{aligned}
\mathcal{L}_{\text {eff }}= & \mathcal{L}_{q 0}+\mathcal{L}_{q M}+\mathcal{L}_{\Sigma \Sigma}+\mathcal{L}_{V V}+\mathcal{L}_{\chi S B} \\
& +\mathcal{L}_{\Delta m_{s}}+\mathcal{L}_{h},+\mathcal{L}_{c},
\end{aligned}
$$

where $\mathcal{L}_{q 0}$ is the free part for massless quarks. The quark-meson interaction $\mathcal{L}_{q M}$ can be written in a chiral $S U(3)$ invariant way as

$$
\begin{aligned}
\mathcal{L}_{q M}= & g_{s}\left(\bar{\Psi}_{L} M \Psi_{R}+\bar{\Psi}_{R} M^{+} \Psi_{L}\right) \\
& -g_{v}\left(\bar{\Psi}_{L} \gamma^{\mu} l_{\mu} \Psi_{L}+\bar{\Psi}_{R} \gamma^{\mu} r_{\mu} \Psi_{R}\right) .
\end{aligned}
$$

The meson self-interaction $\mathcal{L}_{\Sigma \Sigma}$ and $\mathcal{L}_{V V}$ can also be written in a chiral invariant way $[8,9]$. The Lagrangian $\mathcal{L}_{\chi S B}$ generates the nonvanishing masses of pseudoscalar mesons leading to a nonvanishing divergence of the axial currents which in turn satisfy the partial conserved axial-vector current (PCAC) relations for $\pi$ and $K$ mesons. Pseudoscalar, scalar mesons and also the dilaton field $\chi$ obtain mass terms by spontaneous breaking of chiral symmetry. The masses of $u, d$ and $s$ quarks are generated by the vacuum expectation values of the two scalar mesons $\sigma$ and $\zeta$. To obtain the correct constituent mass of the strange quark, an additional mass term $\mathcal{L}_{\Delta m_{s}}$ has to be added. In order to obtain reasonable hyperon potentials in hadronic matter, we include an additional coupling $\mathcal{L}_{h}$ between strange quarks and the scalar mesons $\sigma$ and $\zeta$ [8]. In the quark meanfield model, quarks are confined in baryons by the Lagrangian $\mathcal{L}_{c}=-\bar{\Psi} \chi_{c} \Psi$ (with $\chi_{c}$ given in Eq. (6), below). The Dirac equation for a quark field $\Psi_{i j}$ under the additional influence of the meson mean fields is given by

$$
\left[-i \vec{\alpha} \cdot \vec{\nabla}+\chi_{c}(r)+\beta m_{i}^{*}\right] \Psi_{i j}=e_{i}^{*} \Psi_{i j},
$$

where $\vec{\alpha}=\gamma^{0} \vec{\gamma}, \beta=\gamma^{0}$, the subscripts $i$ and $j$ denote the quark $i(i=u, d, s)$ in a baryon of type $j(j=N, \Lambda, \Sigma, \Xi) ; \chi_{c}(r)$ is a confining potential - i.e., a static potential providing confinement of quarks by meson mean-field configurations. The quark mass $m_{i}^{*}$ and energy $e_{i}^{*}$ are defined as

$m_{i}^{*}=-g_{\sigma}^{i} \sigma-g_{\zeta}^{i} \zeta+m_{i 0}$

and

$e_{i}^{*}=e_{i}-g_{\omega}^{i} \omega-g_{\phi}^{i} \phi$,

where $e_{i}$ is the energy of the quark under the influence of the meson mean scalar fields. Using the solution of the Dirac equation (6) for the quark energy $e_{i}^{*}$ it has been common to define the effective mass of the baryon $j$ through the ansatz:

$M_{j}^{*}=\sqrt{E_{j}^{* 2}-<p_{j c m}^{* 2}>}$,

where $E_{j}^{*}=\sum_{i} n_{i j} e_{i}^{*}+E_{j \text { spin }}$ is the baryon energy and $\left\langle p_{j \mathrm{~cm}}^{* 2}\right\rangle$ is the subtraction of the contribution to the total energy associated with 
spurious center of mass motion. In the expression for the baryon energy $n_{i j}$ is the number of quarks with flavor " $i$ " in a baryon with flavor $j$, with $j=N\{p, n\}, \Sigma\left\{\Sigma^{ \pm}, \Sigma^{0}\right\}, \Xi\left\{\Xi^{0}, \Xi^{-}\right\}, \Lambda$ and $E_{j \text { spin }}$ is the correction to the baryon energy which is determined from a fit to the data for baryon masses.

There is an alternative way to remove the spurious c.m. motion and determine the effective baryon masses. In Ref. [13], the removal of the spurious c.m. motion for three quarks moving in a confining, relativistic oscillator potential was studied in some detail. It was found that when an external scalar potential was applied, the effective mass obtained from the interaction Lagrangian could be written as

$M_{j}^{*}=\sum_{i} n_{i j} e_{i}^{*}-E_{j}^{0}$,

where $E_{j}^{0}$ was found to be only very weakly dependent on the external field strength. We therefore use Eq. (10), with $E_{j}^{0}$ a constant, independent of the density, which is adjusted to give a best fit to the free baryon masses.

\section{HADRONIC SYSTEM}

Based on the previously defined interaction, the thermodynamic potential for hadronic matter is written as

$$
\begin{aligned}
\Omega= & -\sum_{N=p, n} \frac{k_{B} T}{(2 \pi)^{3}} \int_{0}^{\infty} d^{3} \vec{k} \\
& \left\{\ln \left(1+e^{-\left(E_{N}^{*}(k)-\nu_{N}\right) / k_{B} T}\right)\right. \\
& \left.+\ln \left(1+e^{-\left(E_{N}^{*}(k)+\nu_{N}\right) / k_{B} T}\right)\right\}-\mathcal{L}_{M}
\end{aligned}
$$

where $E_{N}^{*}(k)=\sqrt{M_{N}^{* 2}+\vec{k}^{2}}$. The quantity $\nu_{N}$ is related to the usual chemical potential, $\mu_{N}$, by $\nu_{N}=\mu_{N}-g_{\omega}^{N} \omega-g_{\rho}^{N} \rho$. The energy per unit volume and the pressure of the system are respectively $\varepsilon=\Omega-\frac{1}{T} \frac{\partial \Omega}{\partial T}+\nu_{N} \rho_{N}$ and $p=-\Omega$, where $\rho_{N}$ is the baryon density. The mean-field equation for meson $\phi_{i}$ is obtained by the formula $\partial \Omega / \partial \phi_{i}=0$.

Let us now discuss the liquid-gas phase transition. For asymmetric nuclear matter, the system

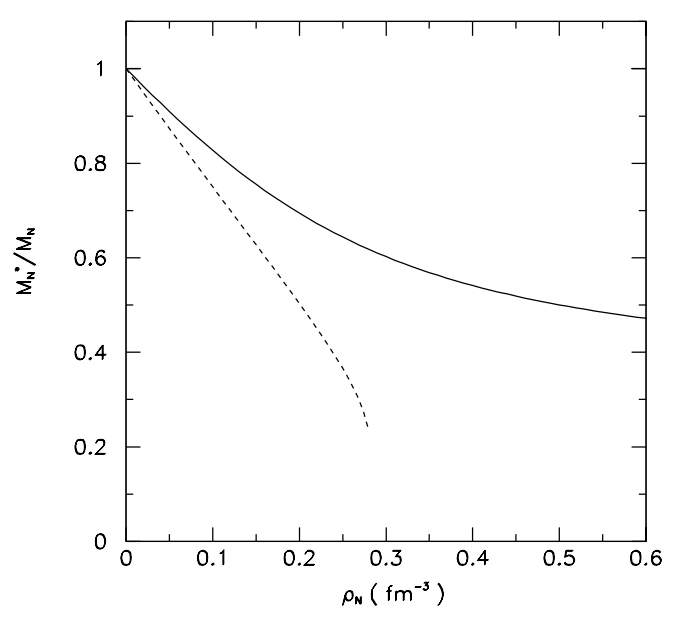

Figure 1. The effective nucleon mass $M_{N}^{*} / M_{N}$ versus nuclear density $\rho_{N}$. The solid and dashed lines are for linear and square root treatments of effective baryon mass, respectively.

will be stable against separation into two phases if the free energy of a single phase is lower than the free energy in all two-phase configurations. This requirement can be formulated as [14]

$F(T, \rho)<(1-\lambda) F\left(T, \rho^{\prime}\right)+\lambda F\left(T, \rho^{\prime \prime}\right)$,

where

$\rho=(1-\lambda) \rho^{\prime}+\lambda \rho^{\prime \prime}, \quad 0<\lambda<1$,

and $F$ is the Helmholtz free energy per unit volume. The two phases are denoted by a prime and a double prime. If the stability condition is violated, a system with two phases is energetically favorable. The phase coexistence is governed by the Gibbs conditions:

$\mu_{j}^{\prime}\left(T, \rho^{\prime}\right)=\mu_{j}^{\prime \prime}\left(T, \rho^{\prime \prime}\right) \quad(j=p, n)$,

$p^{\prime}\left(T, \rho^{\prime}\right)=p^{\prime \prime}\left(T, \rho^{\prime \prime}\right)$

where the temperature is the same in the two phases. For finite nuclei, compared with the case of infinite nuclear matter, the size effect and Coulomb interaction are important. When the Coulomb interaction is considered, the chemical potential for the protons will have an additional term $[15,16]$. 


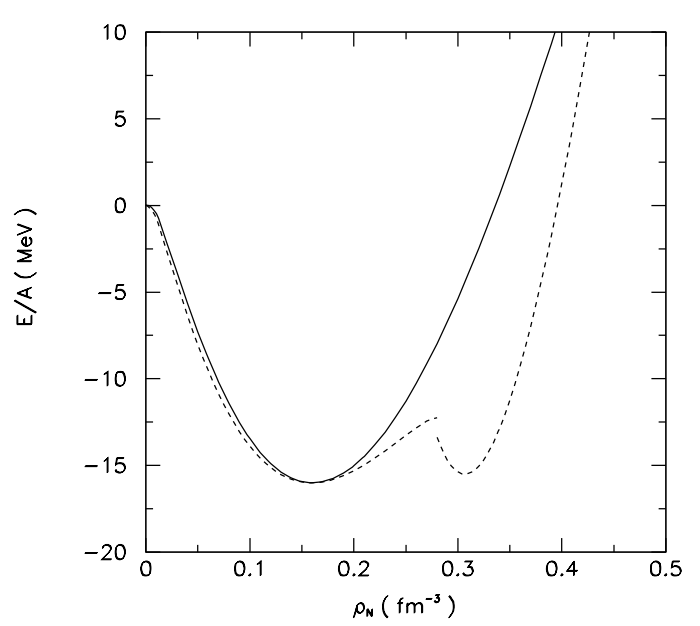

Figure 2. The energy per nucleon E/A versus nuclear density $\rho_{N}$. The solid and dashed lines are for linear and square root treatments of effective baryon mass, respectively.

\section{NUMERICAL RESULTS}

The parameters in this model are determined by the meson masses in vacuum and the properties of nuclear matter which have been discussed in the earlier papers $[8,17]$. We first consider infinite nuclear matter. In Fig. 1, we show the effective nucleon mass divided by the free nucleon mass, $M_{N}^{*} / M_{N}$, versus nuclear density. The effective mass for the square root ansatz decreases faster than that for the linear definition and, as a consequence, at some critical density the effective mass drops to zero. However, in the case of the linear definition, the effective mass decreases slowly at high density and there is no phase transition to a state of chiral symmetry restoration. We plot the energy per nucleon versus nuclear density in Fig. 2. The behavior of the solid line and dashed lines are close when the density is small, say $\rho_{B}<0.2 \mathrm{fm}^{-3}$. Both curves pass through the saturation point of nuclear matter. For the square root ansatz for effective mass, E/A changes discontinuously at the critical density.

For finite systems, we do not adjust the parameters, rather they remain the same as in infinite hadronic matter. The charge density of ${ }^{208} \mathrm{~Pb}$ versus the radius is shown in Fig. 3. The two

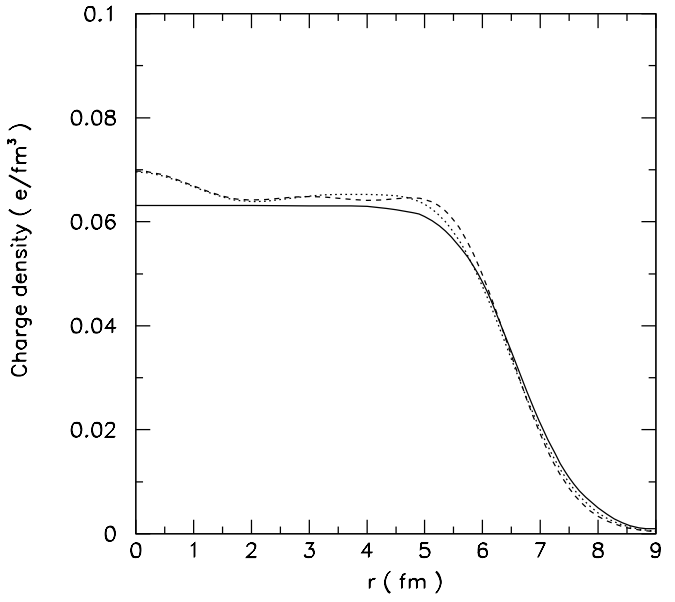

Figure 3. The charge density versus radius for ${ }^{208} \mathrm{~Pb}$. The dashed and dotted lines are calculated with linear and square root treatments of effective baryon mass, respectively. The solid line is from the experimental data.

definitions give similar results. This arises naturally since the nucleon density is around saturation density in the center of finite nuclei and both treatments yield the correct saturation properties of nuclear matter. We plot the proton energy levels of ${ }^{208} \mathrm{~Pb}$ in Fig. 4. The energy levels are qualitatively reproduced. For the square root ansatz for baryon mass, the spin-orbit splitting is quite close to experiment. For example, the splitting of $1 g_{7 / 2}$ and $1 g_{9 / 2}\left(2 d_{3 / 2}\right.$ and $\left.2 d_{5 / 2}\right)$ of ${ }^{208} \mathrm{~Pb}$ is about $4.2 \mathrm{MeV}$ ( $1.6 \mathrm{MeV}$ ) which is close to the experimental value $3.9 \mathrm{MeV}$ ( $1.5 \mathrm{MeV}$ ). For the linear definition of baryon mass, the spin-orbit splitting is smaller. This is because at saturation density, the effective nucleon mass is higher in this definition and the spin-orbit splitting is proportional to the decrease of the effective nucleon mass. However, the smaller spin-orbit splitting can be improved by going beyond the mean-field approximation [18].

We now discuss the liquid-gas phase transition of asymmetric nuclear systems. The $\alpha$ dependence of the critical temperature for infinite nuclear matter is shown in Fig. 5 where $\alpha$ is defined as $\left(\rho_{n}-\rho_{p}\right) /\left(\rho_{n}+\rho_{p}\right)$. The solid and dashed lines correspond to the linear definition and the 


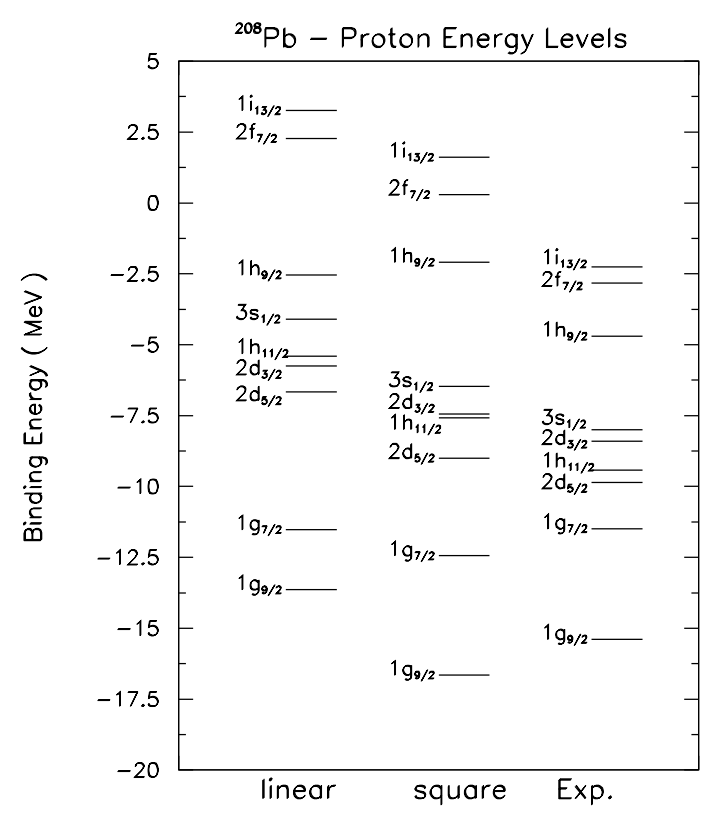

Figure 4. The proton energy levels for ${ }^{208} \mathrm{~Pb}$. The first and second columns are calculated with linear and square root definition of effective baryon mass, respectively. The third column is from the experimental data.

square root ansatz of effective nucleon mass, respectively. The critical temperature $T_{c}$ decreases with increasing $\alpha$. For the square root case, when $\alpha$ is less than 0.2 , the decrease of $T_{c}$ is very small. When $\alpha$ is larger than $0.6, T_{c}$ decreases very fast. If $\alpha$ is larger than 0.88 , the system can only be in the gas phase at any temperature. In the linear case, the critical temperature is $2 \mathrm{MeV}$ larger than that in the square root case. The liquidgas phase transition can occur for nuclear matter with any $\alpha$ if the temperature is lower than the critical temperature.

We show in Fig. 6 the mass number dependence of the limiting temperature, $T_{\text {lim }}$, for nuclei along the line of $\beta$-stability:

$Z=0.5 A-0.3 \times 10^{-2} A^{5 / 3}$.

The solid and dashed lines correspond to the linear definition and the square root ansatz of the effective nucleon mass, respectively. The experimental values obtained recently by Natowitz

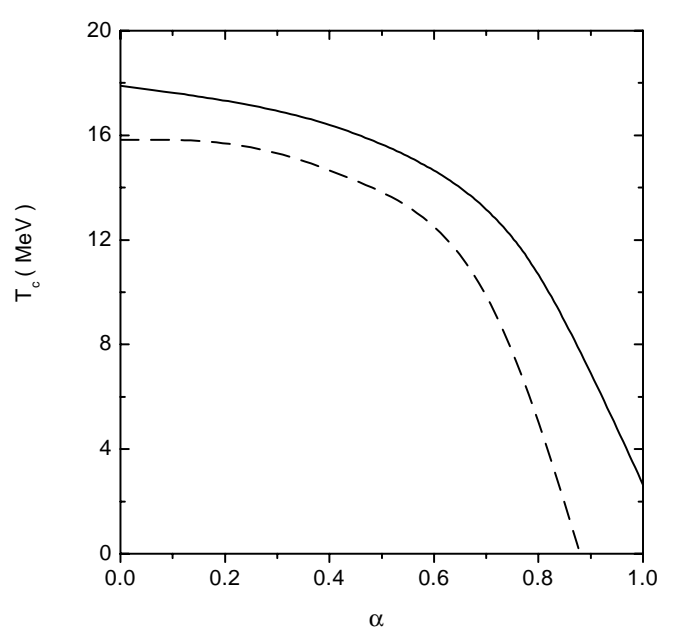

Figure 5. The critical temperature $T_{c}$ versus asymmetry parameter, $\alpha$. The solid and dashed lines are for the linear and square root cases, respectively.

[19] are also plotted in the figure for comparison. The calculated results in this model are in good agreement with the experimental data. The limiting temperature decreases with increasing mass number. This means that when the temperature is higher than the limiting temperature, the heavy nuclei will fragment to light nuclei. Two useful parameterizations of $T_{l i m} / T_{c}$, valid for $10 \leq A \leq 208$, are $\left(T_{l i m} / T_{C}\right)=0.611-$ $0.00193 A+3.32 \times 10^{-6} A^{2}$ (square root case) and $\left(T_{\text {lim }} / T_{C}\right)=0.591-0.00203 A+3.80 \times 10^{-6} A^{2}$ (linear case), which are comparable with that given in Ref. [19].

In summary, we have used an improved treatment of the c.m. motion in calculating the effective, in-medium, baryon mass in an investigation of infinite hadronic matter and finite nuclei within the chiral $S U(3)$ quark mean-field model. The results are compared with earlier results which used the square root ansatz for the effective mass. Both treatments fit the saturation properties of nuclear matter and therefore, for densities lower than the saturation density, these two treatments give reasonably similar results. For high baryon density, the predictions of these two treatments are quite different. There is a phase transition 


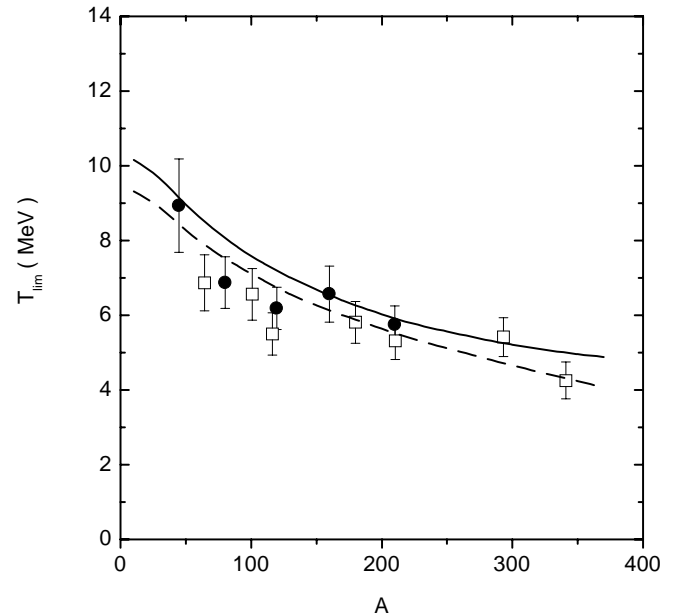

Figure 6 . The limiting temperature, $T_{l i m}$, versus mass number $\mathrm{A}$ of finite nuclei. The solid and dashed lines are for the linear and square root cases, respectively. The points with error bars are from Ref. [19].

of chiral symmetry restoration in the case of the square-root ansatz for baryon mass. Physical quantities, such as effective baryon mass and energy per baryon change discontinuously at the critical density. In the linear case, no such transition occurs. Rather, the effective baryon mass decreases slowly at high density. These qualitatively different behaviors lead to significant differences in the predicted high-density physics.

\section{ACKNOWLEDGEMENTS}

This work was supported by the Australian Research Council and by DOE contract DE-AC0584ER40150, under which SURA operates Jefferson Laboratory.

\section{REFERENCES}

1. J. D. Walecka, Ann. Phys. 83 (1974) 491;

S. A. Chin and J. D. Walecka, Phys. Lett. B 52 (1974) 24.

2. J. Zimanyi and S. A. Moszkowski, Phys. Rev. C 42 (1990) 1416.

3. A. R. Bodmer, Nucl. Phys. A 526 (1991) 703.

4. P. A. M. Guichon, Phys. Lett. B 200 (1988)
235; S. Fleck, W. Bentz, K. Shimizu and K. Yazaki, Nucl. Phys. A 510 (1990) 731; K. Saito and A. W. Thomas, Phys. Lett. B 327 (1994) 9.

5. A. W. Thomas, S. Theberge and G. A. Miller, Phys. Rev. D 24 (1981) 216; A. W. Thomas, Adv. Nucl. Phys. 13 (1984) 1; G. A. Miller, A. W. Thomas and S. Theberge, Phys. Lett. B 91 (1980) 192.

6. H. Toki, U. Meyer, A. Faessler and R. Brockmann, Phys. Rev. C 58 (1998) 3749.

7. W. Bentz and A. W. Thomas, Nucl. Phys. A 696 (2001) 138. M. Buballa, hep-ph/0402234.

8. P. Wang, Z. Y. Zhang, Y. W. Yu, R. K. Su and H. Q. Song, Nucl. Phys. A 688 (2001) 791.

9. P. Wang, H. Guo, Z. Y. Zhang, Y. W. Yu, R. K. Su and H. Q. Song, Nucl. Phys. A 705 (2002) 455.

10. P. Wang, Z. Y. Zhang, Y. W. Yu, Commun. Theor. Phys. 36 (2001) 71.

11. P. Wang, V. E. Lyubovitskij, Th. Gutsche and Amand Faessler, Phys. Rev. C 67 (2003) 015210.

12. P. Wang, H. Guo, Y. B. Dong, Z. Y. Zhang and Y. W. Yu, J. Phys. G 28 (2002) 2265.

13. P. A. M. Guichon, K. Saito, E. Rodionov and A. W. Thomas, Nucl. Phys. A 601 (1996) 349.

14. H. Müller and B. D. Serot, Phys. Rev. C 52 (1995) 2072.

15. A. L. Goodman, J. I. Kapusta and A. Z. Mekjian, Phys. Rev. C 30 (1984) 851.

16. P. Wang, D. B. Leinweber, A.W. Thomas and A. G. Williams, nucl-th/0407057.

17. P. Wang, D. B. Leinweber, A.W. Thomas and A. G. Williams, nucl-th/0404079.

18. T. S. Biro and J. Zimanyi, Phys. Lett. B 391 (1997) 1; G. Krein, A. W. Thomas and K. Tsushima, Nucl. Phys. A 650 (1999) 313; P. A. M. Guichon and A. W. Thomas, nuclth/0402064.

19. J. B. Natowitz et al., Phys. Rev. Lett. 89 (2002) 212701. 